\title{
Combined effects of cold work and chemical polishing on the absorption and release of hydrogen from SRF cavities inferred from resistance measurements of cavity-grade niobium bars
}

\author{
A Dzyuba ${ }^{1,2}$ and L D Cooley ${ }^{1}$ \\ ${ }^{1}$ Superconducting Materials Department, Technical Division, Fermi National Accelerator Laboratory, Batavia, IL \\ 60510, U.S.A. \\ ${ }^{2}$ Novosibirsk State University, Novosibirsk, Russia
}

\begin{abstract}
A series of small fine-grained and single-crystal bars, with strain from $0 \%$ (recrystallized) to $50 \%$, were given different amounts of chemical polishing. Four-point resistivity $(\rho)$ data was used to characterize the electron scattering from dislocations, hydrogen, and any other trace contaminants. As noted by previous studies, annealed $\mathrm{Nb}$ displayed a weak linear increase of $\rho(11 \mathrm{~K})$ with polishing time due to hydrogen absorption, and bulk hydrogen concentration did not exceed $15 \%$ for $200 \mu \mathrm{m}$ metal removed. Cold-worked samples displayed steeper slopes with polishing time (after subtracting resistivity due to strain alone), suggesting that dislocations assist the absorption of hydrogen during polishing. Absorption accelerated above 30\% strain and 100 $\mu \mathrm{m}$ material removal, with room-temperature hydrogen concentration rising rapidly from $2 \%$ up to 5\%. This threshold is significant, since superconducting radio-frequency (SRF) cavities are usually polished as-formed, with $>35 \%$ strain, and polishing removes $>150 \mu \mathrm{m}$ of metal. Resistance jumps between 40 and $150 \mathrm{~K}$, which signal the formation of hydride precipitates, were stronger in cold-worked samples, suggesting that dislocations also assist precipitate nucleation. High-vacuum anneals at $800{ }^{\circ} \mathrm{C}$ for 2 hours, which are known to fully recrystallize cavity-grade niobium and de-gas hydrogen, removed the 40-150 $\mathrm{K}$ jumps and recovered the resistivity increase due to chemical polishing entirely. But, about $30 \%$ of the resistivity increase due to cold work remained, possibly due to residual dislocation clusters. Continued annealing only facilitated the diffusion of surface impurities into the bulk and did not recover the initial 0\% state. Strain, polishing, and annealing thus appear to combine as irreversible paths that change the material. Bearing this in mind, the significant difference in hydrogen uptake between annealed and coldworked samples suggests that annealing SRF cavities prior to chemical polishing could greatly reduce hydrogen uptake and storage in the metal, reducing risk of quality-factor loss. This inverts key steps of the present widely-used cavity processing sequence.
\end{abstract}

\section{Introduction}

Niobium superconducting radio-frequency (SRF) cavity technology enables high-power and high-gradient linear accelerators. An industrial art has become well established, and its present methodology can produce a high yield of cavities at accelerating gradients $E_{A}$ above $25 \mathrm{MV} / \mathrm{m}$ and with quality factors $Q$ above $1 \times 10^{10}$ at $2.0 \mathrm{~K}[1,2,3]$. Almost all cavities are made from recrystallized, finegrained niobium sheet, from which the resonating cells, spokes, and other components are formed by deep drawing and other cold working. The specifications for this starting material [4] are rather stringent, including stipulations that interstitial impurity atom $(\mathrm{C}, \mathrm{N}, \mathrm{O}$, and $\mathrm{H})$ atomic 
concentrations be less than $\sim 200 \mathrm{ppm}$ (30 mass ppm for $\mathrm{O}$, $\mathrm{C}, \mathrm{N} ; 5$ mass ppm for $\mathrm{H}$ ), resulting in a residual resistivity ratio (RRR) of 300 or higher. Assembly of components requires high-vacuum electron-beam welding to maintain material purity. Likewise, many other aspects of the manufacturing process stress the maintenance of high purity and the reduction of sources of contamination.

Once welded, chemical polishing is applied to improve the cavity quality factor $Q$, where either electropolishing (EP) or buffered chemical polishing (BCP) are the most popular techniques. Over a decade ago, it was noted that $Q$ increases with increased material removed up to $\sim 200 \mu \mathrm{m}[5,6]$. This correlation suggests that a "damaged" region of material is removed by polishing, which uncovers pristine metal for the final cavity inner surface. The particular nature of the damage depends partly on cleaning procedures and quality control, since particulate, organic, or inorganic contaminants that are adhered to the surface can easily become incorporated into the near-surface region by mechanical deformation. Even when cleaning is effective, the near-surface can still contain high populations of structural defects and dislocations as a result of friction with forming tools, which can promote increased contamination from external sources [7]. In any regard, aggressive chemical polishing to remove the damaged zone can introduce hydrogen into the metal as a by-product. Cavities loaded with hydrogen in this manner sometimes exhibit "Q-disease", a rapid falloff of $Q\left(E_{A}\right)$ from the onset of accelerating gradient [8, 9, 10]. High-vacuum annealing, e.g. $800{ }^{\circ} \mathrm{C}$ for 2 hours at $<0.1 \mathrm{~Pa}$, de-gases hydrogen from the metal and cures the Q-disease problem [8, 9]. These basic steps, chemical polishing followed by vacuum annealing, have been used for a majority of SRF cavities placed into service or studied scientifically.

It is clear that chemical polishing alters the hydrogen content of the cavity material, and that these changes are undone, to some degree, by high-vacuum annealing. But it is not clear to what degree forming and welding history affects hydrogen activity during subsequent chemical polishing or vacuum annealing. Moreover, many experiments to study chemical polishing in isolation use coupons taken from niobium sheets in the starting annealed condition. By contrast, the deep drawing applied to cavity half cells is significant (sheet specifications call for minimum 35\% elongation) and is rarely recovered before chemical polishing, so the material state actually undergoing chemical polishing in a cavity is quite different than that studied with coupons. Recent experiments [11] to explicitly compare electropolishing behavior of cold-worked coupons to that of annealed coupons suggested that cold work sensitizes niobium to pitting when EP parameters stray from normal settings. Increased chemical activity due to hydrogen and hydrogen bubbles were plausible causes of pits. A follow-up experiment [12] showed that high-vacuum annealing to recover cold work prior to the EP experiment improved this sensitivity. Ref. [12] recommended changing the cavity processing sequence to put annealing ahead of chemical polishing as a preventative measure against pitting. Unfortunately, coupon experiments such as these can only hint at benefits for SRF cavities, because only RF cavity tests give effective data about $Q\left(E_{a c c}\right)$.

The discussion above stresses the avoidance of hydrogen during cavity processing. Hydrogen is a negative agent due to the tendency to form non-superconducting niobium hydrides. Extensive studies since the early 1990s showed that holding a cavity during cool-down in the temperature region around $100 \mathrm{~K}$ can instigate Q-disease in cavities loaded with hydrogen [8-10]. Upon cooling from $300 \mathrm{~K}$, the $\mathrm{Nb}-\mathrm{H}$ phase diagram [13] indicates a loss of solubility of hydrogen in $\mathrm{Nb}$ and the formation of at least 3 or more hydride phases. Very recent in-situ microscopy observations $[14,15]$ of materials specifically taken from cavity fabrication and processing streams confirmed the appearance of very large, $\sim 0.1 \mathrm{~mm}$, hydride precipitates during cooling below $140 \mathrm{~K}$. These observations also noted that the appearance of large precipitates did not depend on strain or details of chemical processing when hydrogen content is sufficiently overpowering.

Curiously, small secondary precipitates appeared along with the large precipitates after cooling below $90 \mathrm{~K}$ in [15], and upon warming and re-cooling these locations became large precipitates at $140 \mathrm{~K}$. The previous locations of large precipitates did not grow as prominently during the second cool-down, and instead small precipitates were co-located with the "skeleton" [15] of the former precipitates. Evidently, there is a subtle interaction between material history and hydrogen transport, where plastic deformation caused by precipitate seeds, mobilization of hydrogen during warming to room temperature, and growth of pre-existing seeds into large precipitates, takes place. Very small precipitates not visible to visible light microscopy in [15] were deduced in the residues of large precipitates, perhaps nucleating along the dislocation networks as seen in [16]. The small precipitates could be suppressed by baking in vacuum at $120^{\circ} \mathrm{C}$ for $48 \mathrm{~h}$, which connects them with loss of $Q$ at high $E_{A}$ as considered in [17]. Among the many discussions of this remedy in the literature, [18] proposes de-binding of hydrogen-vacancy complexes and the initiation of dislocation climb, which could have a profound effect upon hydrogen transport and the ability of hydrogen reservoirs, even those hundreds of micrometers below the surface, to be tapped by growing precipitates.

Small needle-like precipitates can form in intragranular $\mathrm{Nb}$ regions very quickly [19]. Their growth can be slowed by the tendency for $\mathrm{H}$ atoms to become trapped near other interstitial contaminants [20, 21, 22], so ripening should, therefore, be slower in the near-surface region where oxygen is more abundant. Also, since interstitial hydrogen creates an elastic strain on the niobium 
lattice [23], it is energetically favorable to harbor hydrogen along dislocations and grain boundaries, which could make them preferred locations for precipitates. This is a known mechanism of hydrogen embrittlement, for example [24]. By recovering and recrystallizing the metal, hightemperature vacuum annealing not only removes hydrogen but should also dramatically rearrange the locations for hydrogen storage, the network for hydrogen transport, as well as tendencies to nucleate and grow precipitates. The additional electron scattering caused by dislocations and interstitial hydrogen will increase the Ginsburg-Landau parameter, which could lead to flux penetration and dissipation $[18,25]$. On the other hand, some electron scattering can be beneficial for $Q$ [26], so it is desirable to tune carefully the residual amount of dislocations and interstitial hydrogen, or make use of a third agent to provide electron scattering [22, 27-31]. Once collected in a precipitate, hydrogen becomes less effective for electron scattering, so anything that traps hydrogen in interstitial locations is important for cavity quality factor. These are nanostructural aspects that have not been approached before in the context of the metallurgical state of the cavity and its influence on chemical polishing and vacuum annealing.

The exact amount of hydrogen loading as a function of polishing time and material temper has never been systematically investigated, nor has re-arrangement of the sequence above to place annealing ahead of polishing as a preventative measure. Absorption of hydrogen caused by chemical polishing of annealed, well-recrystallized niobium has been broadly studied [32-36], where the strong affinity of niobium for hydrogen has been noted. Left in air at room temperature, freshly annealed niobium will readily absorb hydrogen [36], creating a baseline concentration of between 0.6 and 1.2 at.\% $\mathrm{H}$, depending on other interstitial impurities and the ability of the surface oxide to block diffusion. Thus, de-gassing heat treatments do not produce a permanent state of the metal.

In this paper, we consider chemical polishing (both $\mathrm{EP}$ and $\mathrm{BCP}$ ) and vacuum annealing as is commonly applied to SRF cavities for a series of niobium bars with different degrees of cold work. We use resistivity measurements, in particular at $11 \mathrm{~K}$, to assess small changes in electron scattering due to interstitial hydrogen, dislocations and other structural defects, and other impurities. Here, we rely on the rapid diffusion of hydrogen in niobium at room temperature to re-distribute hydrogen from the surface polishing region throughout the rest of the material. Other potential interstitial contaminants from polishing remain confined to the surface and do not contribute to the resistivity measurements until annealing facilitates their diffusion into the bulk. Also, jumps in resistivity can be observed during temperature sweeps to verify the formation of hydride precipitates. In the next sections, we describe the experiment and present a discussion of the results in the context of changes relevant for SRF cavity technology. We then look more deeply into the plausible material changes that might occur at each stage of the experiment, and we infer how the cavity processing regimen might be improved along the lines of the questions asked earlier in this section.

\section{Experimental procedure}

\subsection{Overview of the experiment}

This sub-section presents a general description of the experiment. Details about the different segments of the experiment are provided in sub-sections that follow. The overall sequence of the experiment is:

1. Many samples were cut from fine-grained annealed sheets or from within a single grain taken from a niobium ingot slice. A first round of resistivity measurements was acquired, denoted by $\rho_{1}$.

2. Groups of samples each were given a certain amount of cold work by elongation in a tensile tester. A second round of resistivity measurements was made for these strained samples, denoted by $\rho_{2}$.

3. Ensembles of samples across the range of strain values (1 unstrained plus 1 from each strain group) were chemically polished together in the same bath. Four such ensembles were processed by EP. Two additional ensembles were processed by BCP. A third round of resistivity measurements was subsequently made for the as-polished samples, denoted by $\rho_{3}$. Some samples were not given polishing as a control group.

4. Strained and polished samples, as well as some samples that were strained but not polished, were collectively annealed under high vacuum in the same oven used to anneal SRF cavities. A fourth round of resistivity measurements was made to the as-annealed samples, denoted by $\rho_{4}$.

5. Most samples were collectively re-annealed as in step (4). A fifth round of resistivity measurements was made, denoted by $\rho_{5}$.

\subsection{Sample preparation}

Most samples were taken from two cavity-grade $\mathrm{Nb}$ sheets taken from a lot actually used to make SRF cavities. The quality certification for the lot indicated compliance with standard specifications, with sampled oxygen concentration lower than 40 mass ppm, carbon and nitrogen each $<30$ mass ppm, and hydrogen concentration lower 3 mass ppm. Except for tantalum (260 mass ppm), metal impurities were below 50 mass ppm. The quality certification indicates RRR of 293 and 297 for the two samples taken; our measurements show RRR of 302 and 305 for the actual sheets used. The fine-grain (FG) sheets were fully recrystallized with a grain size of approximately $35 \mu \mathrm{m}$. 
To better evaluate the role of grain boundaries, additional samples were cut from within a single grain obtained from a niobium ingot slice. These single crystal (SC) specimens were then extracted from the interior of grains $\sim 100 \mathrm{~mm}$ in span, such that entire specimens consisted of a single crystal. No effort was made to identify the crystallographic orientation of the specimens, or align them with certain orientations.

Electrical discharge machining (EDM) was used to cut samples, using a digitally manipulated cutting stage. The dimensions of the samples were $2.5 \mathrm{~mm} \times 2.9 \mathrm{~mm} \times 75$ $\mathrm{mm}$. The first number indicates the width dimension of each cut, which was accurate to an uncertainty of 0.075 $\mathrm{mm}(3 \%)$. This introduces the largest uncertainty in the resistivity measurement. Care was taken to support the soft samples against any bending. The residues of machining were removed by swabbing with a solvent.

The tensile elongation took place with available loads up to $4500 \mathrm{~N}$ using a standard mechanical testing machine. Samples were clamped at both ends, over $66 \%$ of the sample length. This ensured that the deformed region would span the voltage taps in resistance measurements, but not the region where current leads were connected. The elongation rate was kept within recommended limits for tensile testing of pure niobium, $<2 \%$ per minute. The plastic elongations, defined as the incremental increase of length between the clamp points relative to the initial length, were $12 \%, 27 \%, 35 \%$ and $50 \%$.

\subsection{Resistivity measurements}

Resistance was measured by the same 4 point procedure, and with the same apparatus, that is used for quality control of niobium sheets for SRF programs [36, 37] (see also [39]).

One sample is measured at a time. The sample is mounted against a copper block, separated by a thin insulator. In the middle of the copper block is located a thermometer that is specially selected for temperature range studied and calibrated periodically. Clamps with contact area greater than $1 \mathrm{~mm}^{2}$ make current connections to the samples to ensure minimal contact heating. The design of the current contacts produced a contact resistance of less than $10 \mathrm{~m} \Omega$. Spring-loaded pin connectors were used for voltage taps. The strength of the spring was sufficient to slightly deform the sample beneath the pin, ensuring that any surface oxides are penetrated. The voltage taps span the central $25 \mathrm{~mm}$ of the sample, based on a guideline of 10 sample thicknesses in separation from the current contacts to ensure heat deposited at the current contacts will be sunk to the copper block.

The entire assembly of sample, copper block, and all contacts are enclosed in a brass can. The can is attached to a long probe that is lowered vertically into a helium cryostat. Cooling to below $6 \mathrm{~K}$ requires less than 10 minutes without excessive boil-off of liquid helium. The measurement is typically made upon warming, with heat being provided by raising the can progressively higher above the cryogen until the temperature reaches $\sim 200 \mathrm{~K}$ with the can near the top plate of the cryostat. At this point the can is extracted and the measurement is completed with the can in room air. The sample orientation in the cryostat is vertical, which means that vertical thermal gradients in the cryostat could incite temperature gradients along the sample. However, the dimensions of the copper block and its high thermal conductivity are chosen to greatly reduce thermal gradients. A temperature offset also exists between the sample and the copper block due to the thin insulator between them. The superconducting transition of niobium is used to account for temperature uncertainties: When the sample is warmed, heat is conducted to the sample mostly via conduction from the copper block, so the resistive transition associated with the loss of superconductivity is typically measured between 9.4 and 9.6 K, even though the critical temperature of high-purity niobium is $9.23 \mathrm{~K}$. However, because the slope of resistance vs. temperature is low in this temperature range, an uncertainty of $1.0 \mathrm{~K}$ temperature produces an uncertainty of $1 \times 10^{-11} \Omega \mathrm{m}$ for samples with the lowest strain and $3 \times 10^{-11} \Omega \mathrm{m}$ for samples with the highest strain. As we be apparent when the data is discussed later, this uncertainty is about $2 \%$ to $3 \%$. A transition temperature higher than $9.75 \mathrm{~K}$ will invalidate the measurement.

A precision bipolar current source is used to supply current and a sensitive nanovoltmeter is used to acquire voltage signals. The thermal settling time of the apparatus and the filter settings of the voltmeter determine the best parameters for acquiring voltage vs. temperature data, which is converted to resistivity vs. temperature. During initial 10-minute cool down, a current of $90 \mathrm{~mA}$ and $3 \mathrm{sec}$ time interval between measurements were used as a fast method to monitor resistivity changes due to the formation of hydride precipitates. Detailed data were acquired upon warming with a current of $1 \mathrm{~A}$ and $15 \mathrm{sec}$ between measurements. The current was chosen to produce microvolt signals at the transition. Each measurement consisted of a forward and a reverse current pulse (current was off during intervening time), from which data acquisition software subtracted any thermal voltages. The temperature sweep through 6-13 K was typically measured over 10 minutes, and the room-temperature resistivity at 290-296 K was measured over 5 minutes. These parameters we empirically found to minimize noise near the transition, which is related to leveling of thermal gradients. In between these regimes, the warming sweep took place over approximately 30 minutes time.

The acquired $\rho(\mathrm{T})$ curves could be analyzed according to Matthiessen's rule. A clear "knee" in the data was observed at 30-50 K, where electron scattering by phonons takes over from electron scattering by defects and impurities. This provided a clear interpretation of the residual resistivity from the data sets. Moreover, the slope of resistance with temperature below $15 \mathrm{~K}$ was low enough 
to reduce uncertainties due to thermal gradients and temperature offsets to $<3 \%$ as mentioned earlier.

\subsection{Chemical polishing}

Electropolishing took place in a coupon polishing apparatus described previously [11]. An ensemble of samples was polished at the same time to avoid run-to-run variations. Freshly mixed electropolishing solution (the standard 9:1 ratio of $98 \%$ sulfuric to $49 \%$ hydrofluoric acid) was used. Temperature was controlled by recirculating the electrolyte through a heat exchanger immersed in a separate bath. Temperature was held constant at $30{ }^{\circ} \mathrm{C}$. Voltage and current density were adjusted to produce level polishing at a rate of 0.5 $\mu \mathrm{m} \mathrm{min}^{-1}$, which is comparable to cavity polishing. The amount of material removed was determined by weight and thickness measurements before and after EP. Some variation of removal was noticed along the length of the samples, presumably due to variation in local currents or local stirring near the samples.

BCP took place in a beaker using pre-mixed and prechilled acid and an external ice water bath. Temperature was kept below $12{ }^{\circ} \mathrm{C}$. The etching rate was approximately $1.0 \mu \mathrm{m} \mathrm{min}^{-1}$ at this temperature, with the actual amount of material removed being determined by mass and thickness measurements. For both EP and BCP samples, rinsing took place in ultra-high purity water, after which coupons were dried under nitrogen gas and sealed in polyethylene bags to prevent exposure to atmosphere.

\subsection{High-temperature, high-vacuum annealing}

Annealing at $800{ }^{\circ} \mathrm{C}$ for 2 hours took place in the same oven used for cavities, following the established temperature-time and evacuation protocol used for cavities. No cavity or other parts were baked concurrently with the samples. The base pressure of this furnace is below 1.3 $\mathrm{nPa}\left(10^{-8}\right.$ torr). After samples were loaded and the base pressure established, temperature ramp was $10{ }^{\circ} \mathrm{C} \mathrm{min}^{-1}$. During the $800{ }^{\circ} \mathrm{C}$ plateau, pressure in the chamber remained stable at values around $13 \mathrm{nPa}\left(10^{-7}\right.$ torr). Furnace cooling required about $16 \mathrm{~h}$. Room temperature venting was with argon before extracting the samples. All stages of the heat treatment were monitored with a residual gas analyzer.

\section{Results}

\subsection{Results for fine-grained samples}

3.1.1. General trends at $11 \mathrm{~K}$. The residual resistivity at $11 \mathrm{~K}$ is most sensitive to the changes taking place in the samples, so we focus on these data first. After step 3, a matrix of individual data sets is defined, as indicated in Table I. Together, the data show some interesting general trends for the fine-grained samples (see Figure1):

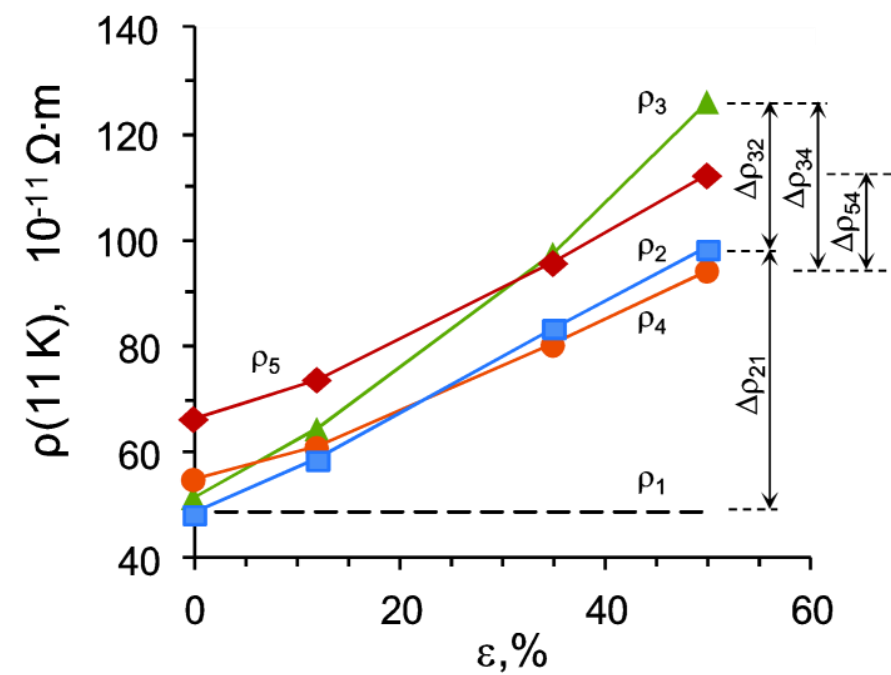

Figure 1. General trends of resistivity at $11 \mathrm{~K}$ are shown, along with a graphical definition of the resistivity changes discussed in the text for the specimen with $50 \%$ strain, as exemplified by the data for the sample series for $180 \mu \mathrm{m}$ material removal by EP.

The horizontal dashed line represents the value of $\rho_{1}$.

1. Resistivity $\left(\rho_{2}\right)$ increases linearly with strain. This result is consistent with earlier studies, e.g. [40], where proportional increase of $\rho$ with dislocation concentration in niobium was established. Using the proportionality constant from [40], dislocation concentrations could be extracted from the $\Delta \rho_{21}=$ $\rho_{2}-\rho_{1}$ values for our samples. These values range from 0 to $40 \times 10^{14} \mathrm{~m}^{-2}$ for 0 to $50 \%$ strain. For comparison, the starting grain boundary density of fine-grained specimens is approximately $10^{9} \mathrm{~m}^{-2}$.

2. For strained samples given chemical polishing, resistivity $\rho_{3}$ is above value of $\rho_{2}$. The amount of incremental increase, $\Delta \rho_{32}=\rho_{3}-\rho_{2}$, increases with the amount of chemical polishing. Since hydrogen uptake is also associated with polishing duration $[33,35]$, it can be inferred that $\Delta \rho_{32}$ is related to hydrogen absorption.

3. The primary source of electron scattering is dislocations, since for all samples the increase of resistivity due to strain, $\Delta \rho_{21}=\rho_{2}-\rho_{1}$, is significantly higher than the additional incremental increase due to chemical polishing, $\Delta \rho_{32}$ (see Figure 1).

4. Resistivity does not increase substantially for the annealed sample with EP time.

5. The amount of incremental resistivity increase, $\Delta \rho_{32}$, also increases with strain present in the sample. That is, strain appears to accelerate the resistivity increase. If the resistivity increase is related to hydrogen uptake, then it can be inferred that the dislocation networks associated with strain assist the uptake of hydrogen. 
Table 1. Measured values of resistivity at $11 \mathrm{~K}$, in units of $10^{-11} \Omega \mathrm{m}$, for specimens taken from a fine-grained niobium sheet. The values prior to polishing are an average of the measurements for each strain group. For the chemically polished samples, the values of $\rho_{3}, \rho_{4}$, and $\rho_{5}$ are shown in sequence.

\begin{tabular}{crrcccc}
\hline \hline $\begin{array}{c}\text { Removal } \\
(\mu \mathrm{m})\end{array}$ & Step & $0 \%$ & $12 \%$ & $27 \%$ & $35 \%$ & $50 \%$ \\
\hline None & $\rho_{1}$ or $\rho_{2}$ & 49 & 56 & 71 & 82 & 96 \\
None & $\rho_{4}$ & 50 & 50 & 56 & 58 & 65 \\
& & & & & & \\
EP: 20 & $\rho_{3}, \rho_{4}, \rho_{5}$ & $49,50,55$ & $56,51,56$ & & $82,60,65$ & $100,66,76$ \\
80 & $\rho_{3}, \rho_{4}, \rho_{5}$ & $49,51,57$ & $57,55,61$ & & $86,67,76$ & $103,74,87$ \\
140 & $\rho_{3}, \rho_{4}, \rho_{5}$ & $50,55,65$ & $61,60,70$ & & $92,72,85$ & $113,86,102$ \\
180 & $\rho_{3}, \rho_{4}, \rho_{5}$ & $51,55,66$ & $65,61,74$ & & $98,80,96$ & $126,94,112$ \\
& & & & & & \\
BCP: 120 & $\rho_{3}, \rho_{4}, \rho_{5}$ & $49,49,58$ & $55,54,63$ & $78,60,72$ & $91,69,82$ & \\
230 & $\rho_{3}, \rho_{4}, \rho_{5}$ & $60,54,67$ & $70,65,87$ & $89,69,109$ & $123,104,128$ & \\
\hline \hline
\end{tabular}

6. Annealing at $800^{\circ} \mathrm{C}$ produces substantial reduction of the resistivity, with $\rho_{4}$ values being significantly lower than the corresponding $\rho_{3}$ values for the same sample. Values of $\rho_{4}$ do not return to the value of $\rho_{1}$, however, and often lie close to the value of $\rho_{2}$, indicating that removal of strain is not complete and that some contaminants may have been introduced during the anneal.

7. Repeating the annealing step produces an additional resistivity increase, with $\rho_{5}>\rho_{4}$ in all cases. This may be due to the further penetration of interstitial contaminants, which enter from the surface, into the bulk. Since the incremental change $\Delta \rho_{54}=\rho_{5}-\rho_{4}$ is always positive, there is no evidence for further removal of either dislocations or hydrogen by the second annealing. Also, since $\Delta \rho_{54}$ is somewhat higher for samples with higher strain, it is plausible that other contaminants also use the residual network of dislocations to move from the surface (where the effect on measured resistivity is small) into the metal bulk.

3.1.2. Estimation of hydrogen uptake and removal. Hydrogen uptake can be estimated from the ratio $\Delta \rho_{32} / \rho_{2}$. Westlake [41] noted that the incremental increase of resistance $\Delta R / R$ at $298 \mathrm{~K}$ shared a linear relationship with the content of hydrogen extracted by vacuum from a loaded sample. Here, $R$ is the resistance of the unloaded sample, and $\Delta R$ is the difference between resistance values before and after hydrogen loading. Westlake's proportionality constant was $[\mathrm{H} \%] \approx 20 \Delta R / R$. Isagawa [36] noted a similar linear slope at $285 \mathrm{~K}$ and $77 \mathrm{~K}$, and in addition obtained data at $9.5 \mathrm{~K}$ for annealed $\mathrm{Nb}$, showing $[\% \mathrm{H}] \approx 8.5 \Delta R / R$. Isagawa also indicated an intercept of $0.65 \% \mathrm{H}$, which we take into account here. The uncertainties noted by Isagawa introduce the main uncertainties in our hydrogen estimate. If we assume that these results are applicable to cold-worked samples as well as annealed samples (we will discuss the validity of this assumption shortly), then by placing our values of $\Delta \rho_{32} / \rho_{2}$
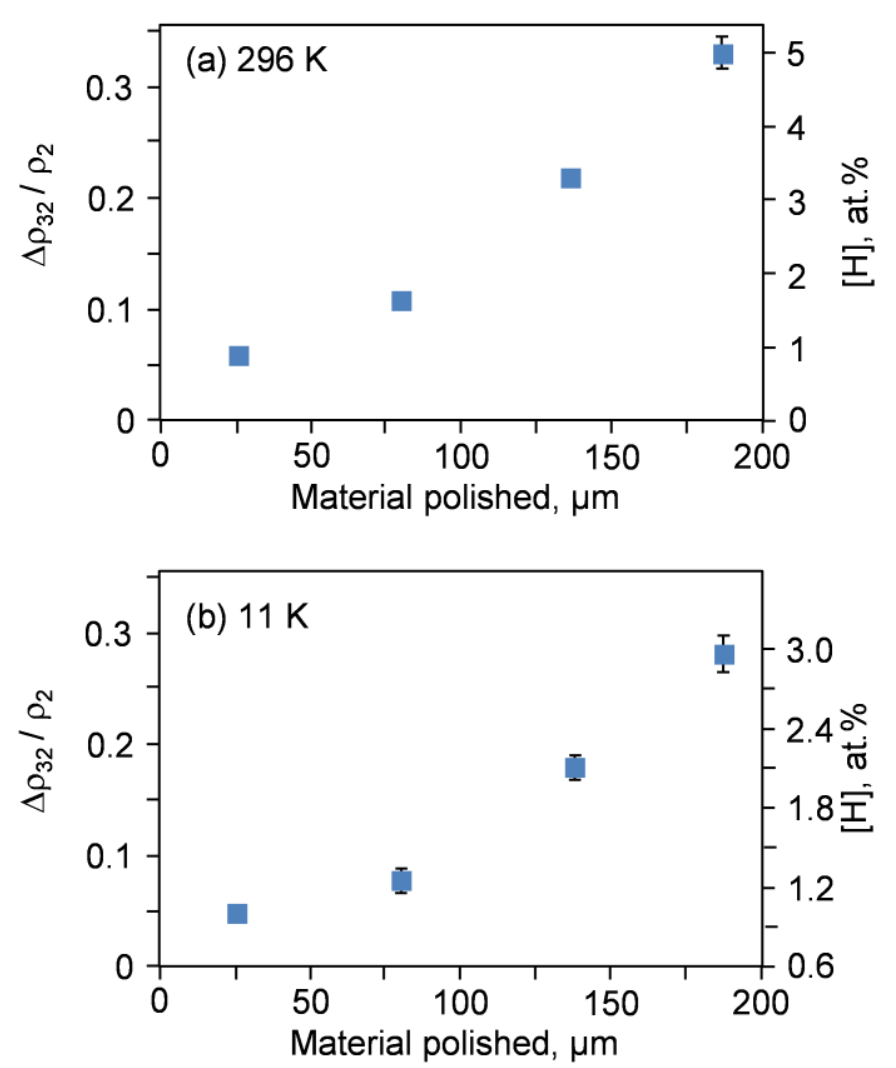

Figure 2. Plots describing the hydrogen content converted from the observed resistivity change are shown. In (a), data acquired at room temperature are plotted, and the hydrogen concentration indicated on the right vertical axis is taken from the linear trend noted by [41] and subsequently by [36]. In (b), data acquired at $11 \mathrm{~K}$, which was summarized in Table 1, are plotted, and the hydrogen concentration indicated on the right vertical axis is based on the linear trend noted by [36] at $9.5 \mathrm{~K}$. The intercept noted in [25] is also included here. 


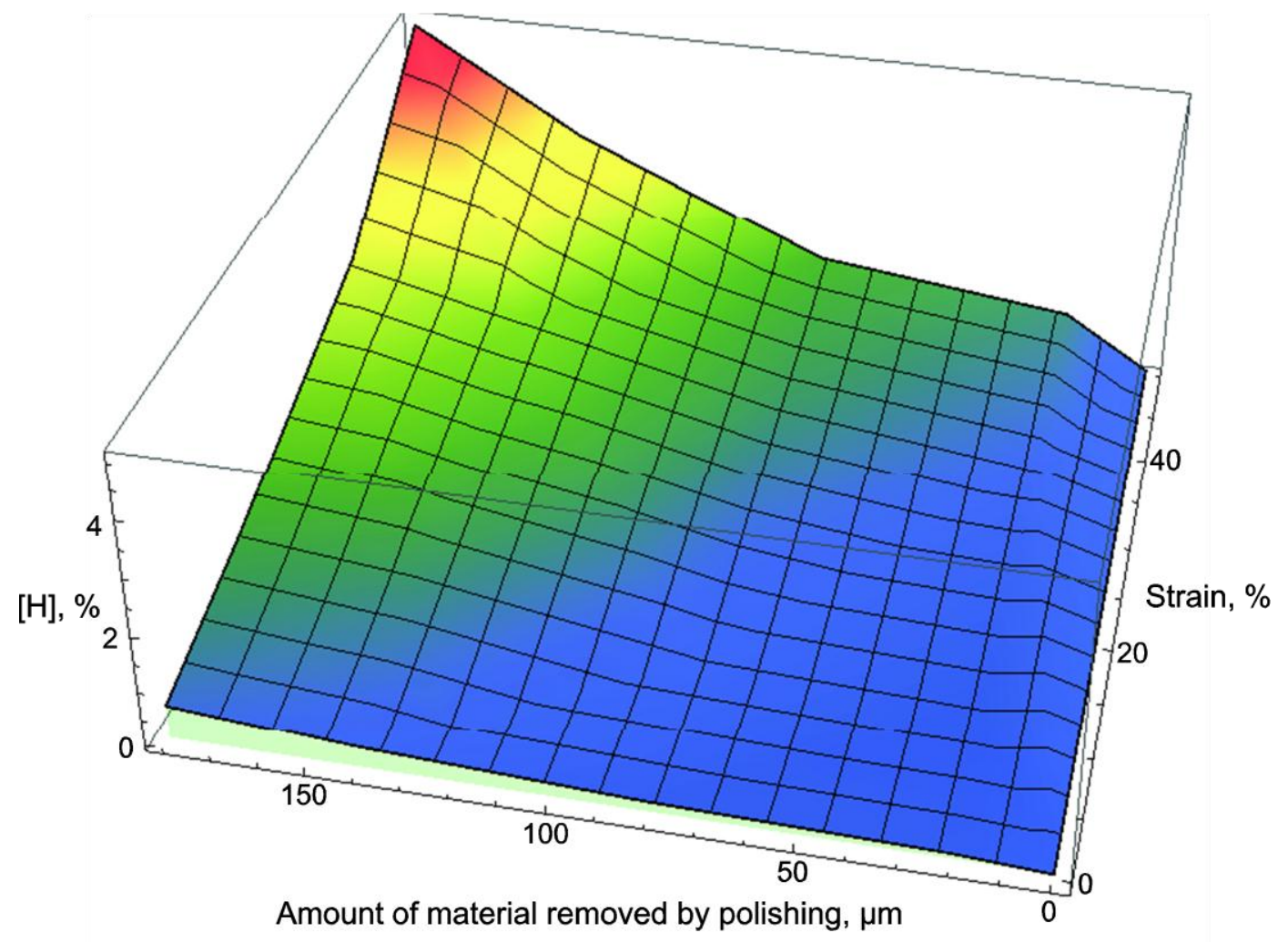

Figure 3. A surface plot estimating the amount of hydrogen absorbed by specimens, as functions of material removal and strain, is shown. As described in the text, the hydrogen content was estimated from $\Delta \rho_{32} / \rho_{2}$ at room temperature using the linear relationship found in [36] and [41].

onto the slope identified by Isagawa, the percentage of hydrogen can be estimated. These ratios and their conversions to $[\mathrm{H} \%]$ are shown in Fig. 2 for resistivity data acquired at $298 \mathrm{~K}$ as well as at $11 \mathrm{~K}$. Somewhat less hydrogen, e.g. $3 \%$ vs $5 \%$ for $50 \%$ strain and $180 \mu \mathrm{m}$ removal, is indicated at $11 \mathrm{~K}$ than at $298 \mathrm{~K}$, which we attribute to hydrogen being confined in precipitates, as previously asserted by Isagawa.

The analysis above can be extended to all of the finegrained specimens, which results in an overall surface relating [H\%] extracted from $\Delta \rho_{32} / \rho_{2}$ to strain and polishing time. This is presented in Fig. 3. Here, we only present the data for EP and we use the $\Delta \rho_{32}$ and $\rho_{2}$ values at room temperature, close to the temperature where actual cavity polishing is performed. Notice that this surface increases steeply for strain or polishing combinations above the contour corresponding to $\sim 1.5 \% \mathrm{H}$ and connecting $\sim 80 \mu \mathrm{m}$ polished at $50 \%$ strain to $\sim 180 \mu \mathrm{m}$ polished at $20 \%$ strain. The coordinates for cavity cells, about $40 \%$ strain and $150 \mu \mathrm{m}$ material polished, lie above this contour, indicating that dislocations should significantly assist hydrogen uptake in typical SRF cavities. On the other hand, for strain or material removal below the $\sim 1.5 \% \mathrm{H}$ contour connecting $20 \mu \mathrm{m}$ polished, $40 \%$ strain, and $150 \mu \mathrm{m}$ polished, $0 \%$ strain, there is hardly any change compared to the original annealed sample. Here, it is noteworthy that many cavities receive a final "light" EP that removes $\sim 20 \mu \mathrm{m}$ of material, and this is performed after the cavity has been annealed. This processing step would take place at or below this lower contour. Also, the strong slope at $\sim 150 \mu \mathrm{m}$ material polished suggests that removing strain before heavy polishing can be an effective way to limit hydrogen uptake.

Based on other studies of niobium (as summarized in [7]), the heat treatment given is expected to fully recover the metal and produce polygonal, equiaxed grains. This is equivalent to the starting material condition. However, in no case does $\rho_{4}$ return to the starting condition $\rho_{1}$, and a finite residue $\Delta \rho_{41}=\rho_{4}-\rho_{1}$ remains. There are 2 primary contributions to this residue: pockets of retained dislocations [7], and interstitial impurities diffusing inward from the surface. The residual cold work alone is indicated by $\Delta \rho_{24}=\rho_{2}-\rho_{4}$. Notice that $\Delta \rho_{24}$ is an increasing function of strain in table 1 . Evidently, $800{ }^{\circ} \mathrm{C}$ does not provide enough thermal energy to produce a recrystallization rate fast enough to remove all residues of strain, despite the availability of energy stored in the deformed material. The residue after simultaneous recovery and de-gassing can be expressed by $\Delta \rho_{34}=\rho_{3}-$ $\rho_{4}$. Interestingly, $\Delta \rho_{34}$ shows weak if any dependence on the amount of, or type of, chemical polishing, and the values scatter around the value of $\Delta \rho_{24}$. Values of $\Delta \rho_{34}$ are plotted as a function of material removal by EP in Fig. 3(a) for the different groups of cold work. This emphasizes the effective de-gassing of the anneal. 

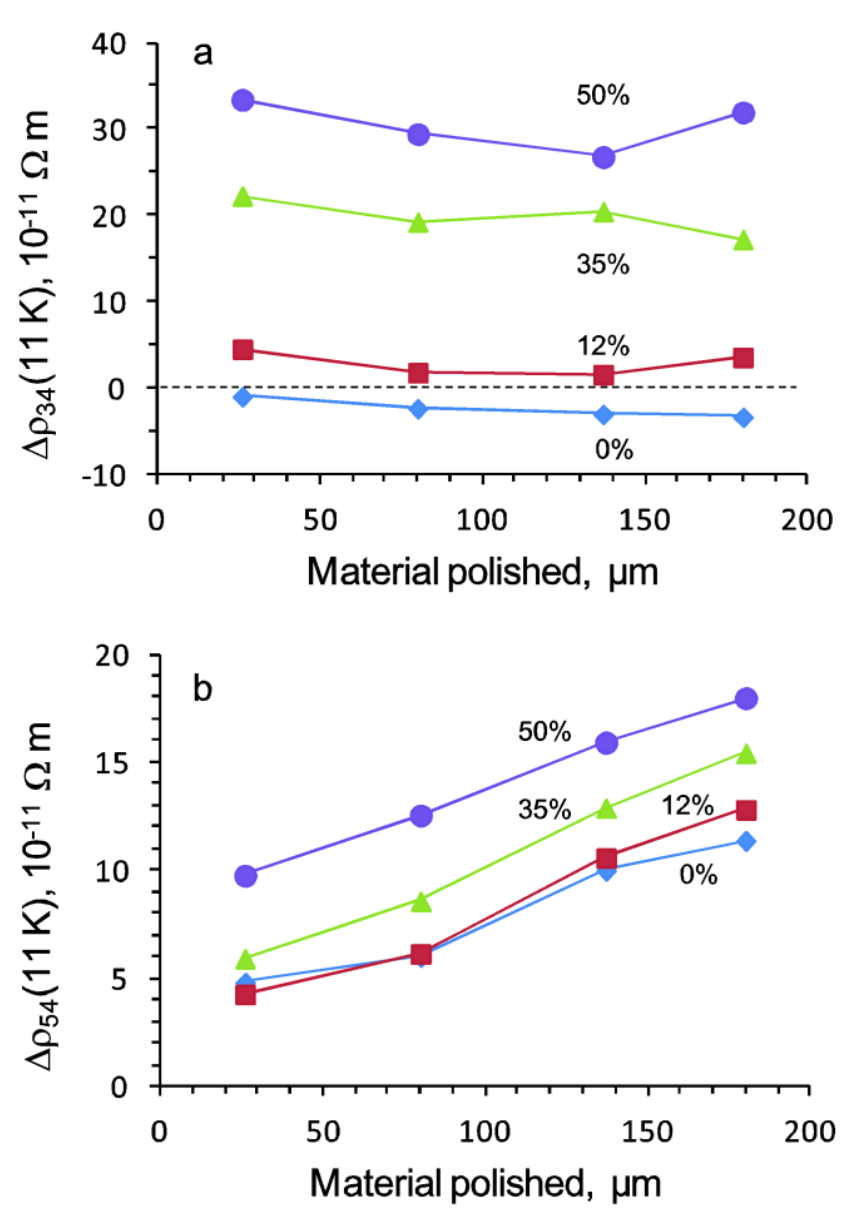

Figure 4. Changes in resistivity at $11 \mathrm{~K}$ during recovery are described in these plots. In plot (a), the difference between stages 3 and 4 of the experiment is plotted as a function of the amount of chemical polishing by EP, for different groups of strain. The negative values for the $0 \%$ data indicate higher resistivity at stage 4 than at stage 3 , as discussed in the text. In plot (b), the changes in resistivity between stages 5 and 4 are plotted as a function of material removed by EP for the different strain groups.

For $0 \%$ and $12 \%$ strain, the electron scattering by dislocations is less than or comparable to that caused by impurity atoms. Here, $\Delta \rho_{34}$ is slightly negative, which is due to diffusion of trace contaminants from the surface into the bulk. We surmise that the large cavity oven, which is not designed or intended for annealing small samples, produced an environment local to the samples richer in contaminants than evidenced by the RGA data. It is also plausible that dissolution of the surface oxide contributes interstitial contaminants at the level of scattering seen. This trend continues for further annealing, with $\rho_{5}>\rho_{4}$ for all samples. In this case, the difference $\Delta \rho_{54}=\rho_{5}-\rho_{4}$ has weak dependence on chemical polishing time. This is shown in Fig. 3(b). Here the values $\Delta \rho_{54}$ for the samples which received $\mathrm{BCP}$ were substantially higher than the samples which received $\mathrm{EP}$.

3.1.3. Temperature-dependent resistivity data 11-300 K. The resistivity curves acquired over the entire temperature range are also instructive. The temperature-dependent data sets $\rho_{1}(T)$ were virtually identical since the samples all came from a single sheet of niobium. Likewise, for each value of tensile strain $\varepsilon$, similar values of $\rho_{2}(T)$ was recorded from sample to sample.

The $\rho_{3}(T)$ data for the most heavily polished samples showed changes in the slope of resistivity between 50 and $150 \mathrm{~K}$. By contrast, neither the $\rho_{2}(T)$ nor the $\rho_{4}(T)$ data display such changes, and also the $\rho_{3}(T)$ data for mild polishing did not display noticeable slope deviations. This suggests that the formation of hydride precipitates, which is known to occur around $100 \mathrm{~K}[13,42]$, is the cause of the resistivity changes in the $\rho_{3}(T)$ data for the heavily polished samples. These transitions are most evident when both $\rho_{3}(T)$ and $\rho_{4}(T)$ data are plotted together, as shown in Fig. 4(a) and Fig. 4(b).

Evidently, the formation of hydride precipitates contributed significant additional scattering between 50 and $150 \mathrm{~K}$ for stage 3 of the experiment. This is emphasized in Fig. 4(c). Isagawa [36] and Westlake [41] both reported a drop of resistance upon the formation of precipitates, which occurred after the niobium metal became saturated with hydrogen. In their annealed samples, the ordering of hydrogen in precipitates was thought to result in electrical pathways through conductive hydrides with overall conductivity comparable to that of the clean niobium metal, in contrast to the higher scattering for random solution of hydrogen in niobium prior to ordering. In our samples, electron scattering is dominated by dislocation networks. Since the deviation in Fig. 4(c) is higher for the samples with higher strain, we speculate that hydride precipitates form along the dislocation network and enhance their scattering fields. Location of hydride precipitates along such networks is consistent with mechanisms of hydrogen embrittlement in metals [24, 43] as was mentioned in the introduction.

\subsection{Single-crystal results}

The presence of grain boundaries should affect the removal of dislocations during the annealing and recovery. In order to investigate interaction between grain boundaries and hydrogen, single-crystal bars were submitted to the same experiment, using strains of $0 \%, 12 \%$ and $30 \%$ and applying EP for 150 and 405 minutes $(80 \mu \mathrm{m}$ and $180 \mu \mathrm{m}$ material removed). The results are compared to finegrained bars in Figure 6. Approximately the same rate of hydrogen uptake was seen, as shown in plot (a) from the resistivity increase $\Delta \rho_{32} / \rho_{2}$. We speculate that the offset for the $0 \%$ sample indicates an amount of work stored in the single crystal samples due to the ingot slicing, which was not recovered prior to chemical polishing. The RRR of the single crystals, 221, was slightly lower than that of the fine-grained bars, but not so different as to impart a large contribution from other interstitial impurities. It is also possible that the cutting method itself introduced hydrogen. If true, the value of $\rho_{2}$ should then include effects of 

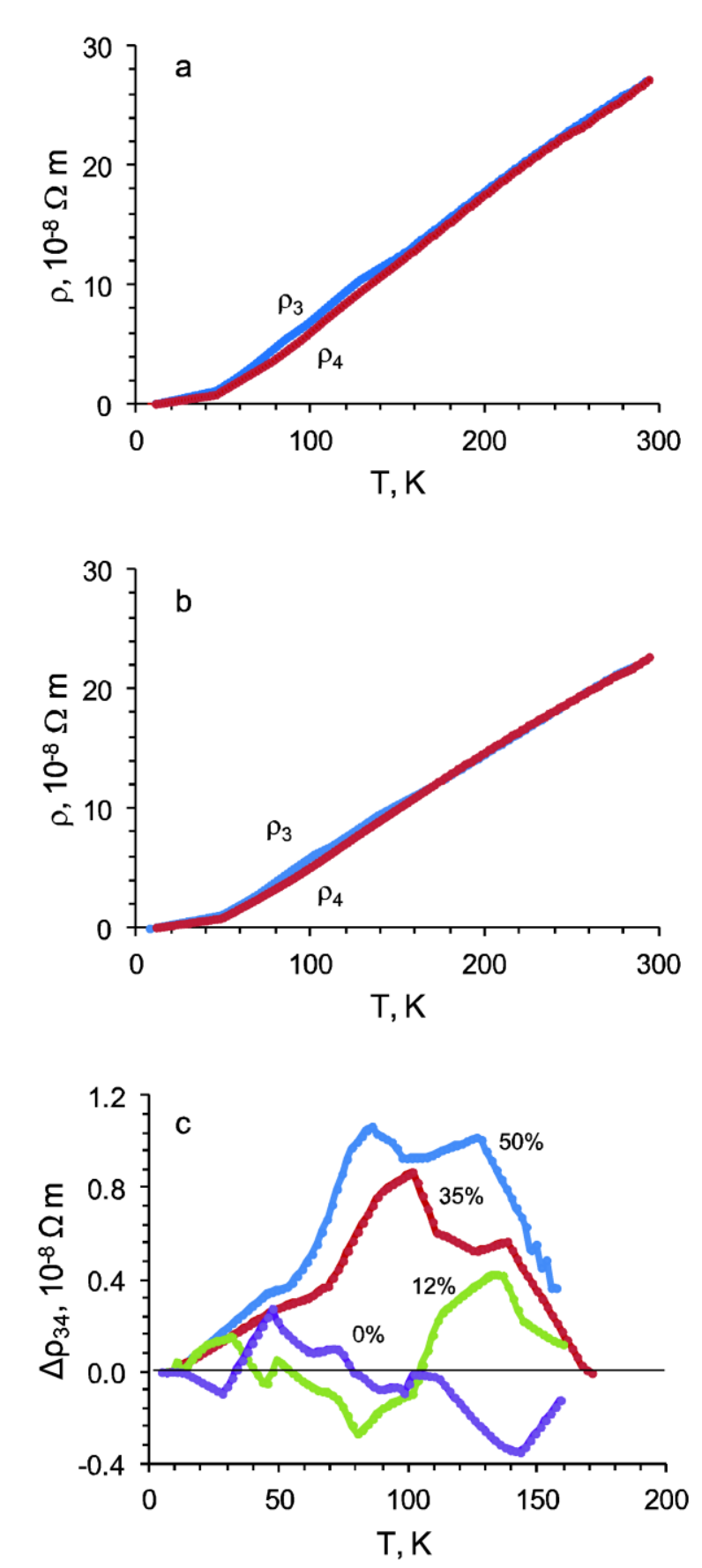

Figure 5. Stage 3 and stage 4 resistivity are plotted together as a function of temperature for different strain values and for 180 $\mu \mathrm{m}$ material removal by EP in plots (a) for $50 \%$ strain, and plot (b) for $35 \%$ strain. In plot (c), data were subtracted to emphasize the changes seen in the temperature range where hydride precipitates are known to form.

hydrogen loaded into the sample by cutting. Upon annealing, the change in resistivity $\Delta \rho_{32}$ could then be higher because hydrogen loaded by the cutting technique, in addition to hydrogen loaded by chemical polishing, could be removed from the sample.

It is interesting, however, that the single crystal specimens have lower slopes of resistivity increase with strain, and different rates of recovery, than the fine-grain specimens do. Different resistivity increase with strain was noted in [39], and the slopes in plots (b) and (c) of Fig. 5 are consistent with those earlier data. The different slopes may reflect the contribution of grain boundaries in the finegrain specimens to the resistivity. Hydrogen residing in grain boundaries can then increase their electron scattering. The changes observed upon annealing are more sluggish for SC bars than the FG specimens. In Fig. 5(b) for single crystals that received $80 \mu \mathrm{m}$ of polishing, the stage 4 resistivity returns to the stage 2 value, but does not fall below the stage 2 trend. Whereas, the recovery for the corresponding fine-grained specimen resulted in a stage 4 resistivity well below the stage 2 trend (the reader should take note of values in table 1 not plotted in Fig. 5(b)). In Fig. 5(c) for single crystals that received $180 \mu \mathrm{m}$ of polishing, hardly any reduction in resistivity is observed after the anneal. Thus, while grain boundaries with hydrogen appear to add electron scattering in FG samples, they also appear to permit more effective removal of scattering locations, probably dislocations, by the anneal.

\section{Discussion}

It is well known that dislocation networks can assist diffusion of atoms, particular interstitial impurity atoms $\mathrm{H}$, $\mathrm{C}, \mathrm{N}$, and $\mathrm{O}$. Diffusion short-circuiting by dislocation networks has been observed for many metals, both single grain and polycrystalline [44-46]. These general qualities apply to niobium, where hydrogen uptake appears to be assisted by the dislocation networks produced during cold work.

The appearance of strain-polishing coordinates associated with an upturn in hydrogen absorption, Fig. 2, is an important result. Since SRF cavities combine a strain state and a material polishing requirement that locates above the upturn, the propensity for hydrogen absorption is higher than might be predicted from coupon studies. Solutions to this problem are to reduce cold work, reduce chemical polishing, or reduce both. That is, by applying annealing ahead of chemical polishing, significant reduction of hydrogen uptake can be achieved, perhaps to the point where the usual vacuum de-gassing anneal can be omitted altogether. Perhaps the material removal requirement can be reduced as well. This could be an important cost-savings route: Annealing requires modest resources, since it can be done by batch and unattended by workers. Whereas, chemical polishing is hazardous and requires constant attention of highly trained specialists, due to the need for hydrofluoric acid to solvate niobium salts.

A second interesting result is the agreement in resistivity changes between different types of chemical polishing. The data for BCP in Table 1 align quite well with the data for EP, both in the magnitude of resistivity changes and in the quantitative trends. While there should be differences in the availability of hydrogen based on the differences in chemical processes [33], evidently these differences do not show up in this experiment. 

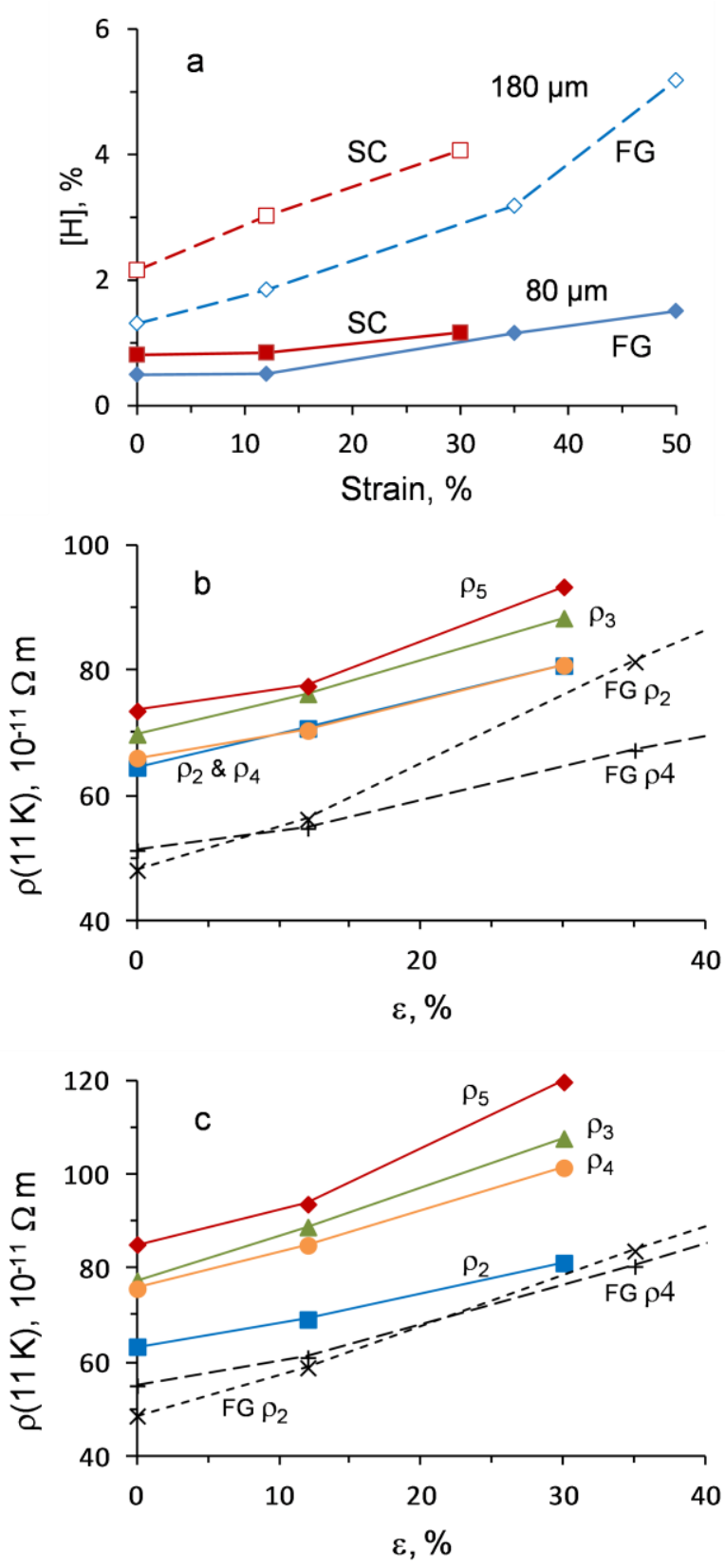

Figure 6. Hydrogen absorption at room temperature deduced from the change in resistivity is compared for fine grain (FG) specimens and single crystal (SC) crystals for two different amounts of EP in plot (a). The conversion from [41] was applied, as used for Fig. 2. Unknown deformation due to ingot slicing to obtain the $0 \%$ SC samples was not removed by annealing prior to chemical polishing. Plots (b) and (c) repeat the sequence shown in Fig. 1, for $80 \mu \mathrm{m}$ and $180 \mu \mathrm{m}$ material removed, respectively. Also plotted are the corresponding stage $2(\times)$ and stage $4(+)$ data for the FG samples with the same material removal.

A third important result is evidence that the effects of hydrogen are additive to those of dislocation networks. This immediately suggests that a primary advantage of the $800{ }^{\circ} \mathrm{C}$ vacuum anneal is to greatly reduce the dislocation density. Here, grain boundaries may play an important role as sinks for dislocations during annealing, which helps to reduce the dislocation population for a given anneal time.
By contrast, single crystals do not have this sink, and would tend to retain a higher population of dislocations for the same heat treatment parameters.

A fourth result is the increase of resistivity resulting from the transition through the 90-140 K regime where hydride precipitates form. Strong drop of resistivity was noted by Isagawa [36] and Westlake [41] when precipitates formed, for samples in a starting annealed state. Isagawa and Westlake both noted that, above $140 \mathrm{~K}$, hydrogen starts as a random solid solution in niobium metal. Electron scattering is provided by the strain field around the interstitial atoms [47, 48] and some charge transfer from the $\mathrm{H}$ atom to the $\mathrm{Nb}$ lattice $[47,49]$. Upon cooling and precipitate formation, these strain fields are largely removed, leading to resistivity drop. In our experiment, dislocations appear to provide pipelines for rapid hydrogen penetration of the interior. It is possible that hydrogen then diffuses outward from these networks into the surrounding niobium metal. However, strain energy increases as interstitial hydrogen enters the niobium lattice [47]. This leads us to believe that, in cold-worked samples above $140 \mathrm{~K}$, dislocations instead provide stable traps for hydrogen, which results in a swelling of the dislocation strain fields with increasing hydrogen content as indicated by neutron scattering studies [50-53]. Then, in view of [15], we surmise that the resistivity increase in the 90 $140 \mathrm{~K}$ region may be due to the formation of small precipitates all along the dislocation network.

Earlier, we applied the initial slope found by Isagawa to ascertain the hydrogen concentration associated with the resistivity change $\Delta \rho_{32} / \rho_{2}$. Since the dislocations are the primary electron scattering sites in the cold-worked metal, and since the enhancement of scattering by hydrogen absorption appears to arise from increase in the strain field for either annealed or cold-worked samples, we feel justified in extrapolating hydrogen content from the $\Delta \rho_{32}$ data using the initial slope found by Isagawa. Actual vacuum extraction measurements of hydrogen content were not attempted to validate this approach further. Vacuum extraction was used by Isagawa.

It is also interesting that signatures of hydride precipitates showed up in sample tests (Fig. 5), where the time spent in the incubation temperature range was several minutes. This is significantly shorter than the long holding time at $100 \mathrm{~K}$ used to test for Q-disease in cavities, but it is consistent with direct observations $[14,15,19]$. If our discussion point above, that hydrogen remains strongly associated with dislocation networks upon cooling, is valid, then this discrepancy of precipitation rate is curious. The reasonable explanation might be the importance of the size of nucleated hydride. The resistivity jumps may be associated with small hydrides nucleated instantly along the dislocation networks. Whereas, the large precipitates connected with Q-disease requires growth over time. Ref. [15] reported a logarithmic growth rate with a time exponent of 0.8 . 
We note that the cavity tests sample only the nearsurface region of metal, whereas resistivity measurements probe the bulk and are weakly sensitive to the surface impurities. On the other hand, we think similar processes of hydride precipitation are occurring both at the surface and within the bulk, so the insights gained from the resistivity measurements are important for understanding electron scattering near the cavity surface. We were surprised by two results in [15], first that the sites where large precipitates formed when first cooled to $140 \mathrm{~K}$ did not re-emerge as large precipitates upon warming to room temperature and cooling a second time; and, second, that a secondary precipitate stage occurred at $90 \mathrm{~K}$ which, upon warming and re-cooling, became the locations for primary precipitation at $140 \mathrm{~K}$. The authors suggested that dislocations trap hydrogen in Cottrell atmospheres around them, which disperses hydrogen in many small precipitates such as the "skeletons" of the large precipitates from the first cool-down. We agree with this idea, since our resistivity measurements indicate an increase of electron scattering between 90 and $140 \mathrm{~K}$ that depends on the amount of strain. The secondary precipitation phase could then indicate phases that form more slowly with the residual hydrogen content remaining after the $140 \mathrm{~K}$ stage. Ref. [16] observed at least 3 different hydride phases by cooling in-situ in an electron microscope to $77 \mathrm{~K}$, so it is also possible that the $90 \mathrm{~K}$ stage involves precipitates with different structure and composition than $\beta-\mathrm{NbH}$. Evidently, warming to room temperature re-distributes hydrogen in the specimen, which then permits the $90 \mathrm{~K}$ seeds to grow at $140 \mathrm{~K}$ during the second cool-down. We do not know to what extent the dislocation network affects this hydrogen re-distribution. Since the "blisters" left behind by large precipitates appear to be regions of plastic deformation, there must be new dislocations formed. Yet, the new dislocations are not capable of pinning down the same amount of hydrogen during the second cool-down. Perhaps this is because the Cottrell clouds are much smaller at room temperature, and the dislocation network instead behaves like a pipeline for hydrogen transport. These are important questions to clarify with future studies, since dislocations also connect the surface with hydrogen sources and sinks located deeper in the bulk and away from the RF currents.

In our experiment, a second annealing time was required to diffuse surface impurities deep enough into the bulk to affect $\rho_{5}$, so we conjecture that there is a zone of increased contamination by oxygen and perhaps carbon and nitrogen just below the surface. The positive slope in Fig. 4(b) suggests that contaminants use the residues of the dislocation network. Vacancies can also be injected by the EP process $[54,55]$, and they should be included in this zone. All of these additions create traps for, and complexes with, for hydrogen atoms [20, 56]. Since hydrogen diffusion should then be slowed by trapping in this zone, growth of any precipitates that nucleate, even those nucleated on dislocations, occurs much more slowly if at all. Re-supply of hydrogen to the surface from the deeper bulk might not overcome these trapping effects. With this in mind, repetition of these measurements with lower grades of niobium, which have 10 times higher interstitial impurity concentration, would be interesting if the resistivity changes due to hydrogen uptake can be detected above the high background scattering.

\section{Conclusions}

An experiment was conducted to investigate the combined effects of cold work and chemical polishing on SRF cavity-grade niobium bars. Resistance measurements, especially at $11 \mathrm{~K}$, were found to be sensitive to the different material states and were therefore used to probe quantitatively these effects as functions of strain (tensile elongation) or polishing time. Both $\mathrm{EP}$ and $\mathrm{BCP}$ were investigated, and strain varied from $0 \%$ (as-received annealed condition) to $50 \%$.

While cold work provided a strong increase of the $11 \mathrm{~K}$ resistivity approximately proportional to the amount of strain, additional increase of resistivity at $11 \mathrm{~K}$ was observed after chemical polishing. Based on previous work on annealed coupons, the change in resistivity could be associated with the concentration of hydrogen absorbed during polishing. Up to 5\% hydrogen was absorbed in the most heavily polished samples at room temperature. The uptake of hydrogen was not linear, and instead displayed an upturn for polishing-strain combinations between $80 \mu \mathrm{m}$ polished at $50 \%$ strain to $180 \mu \mathrm{m}$ polished at $20 \%$ strain. This contour represents approximately $2 \% \mathrm{H}$ absorbed during EP. Importantly, the coordinates for SRF cavities lie above this threshold for accelerated absorption, being $150 \mu \mathrm{m}$ or greater material polished and $35 \%$ or more strain imparted during deep drawing of half cells (the local strain varies with location). Since SRF cavities are chemically processed without removing the cold work during forming operations, it can be concluded that they are rather highly susceptible to hydrogen absorption. Furthermore, the present experiment did not uncover strong differences between EP and $\mathrm{BCP}$, and both processes were found to contribute hydrogen according to the strain state of the specimen.

By contrast, uptake of hydrogen was very low below polishing-strain combinations of $20 \mu \mathrm{m}$ polished, $40 \%$ strain, and $180 \mu \mathrm{m}$ polished, $0 \%$ strain, with less than $0.5 \%$ $\mathrm{H}$ absorbed. This is comparable to the amount of hydrogen that clean metal can absorb from ambient environmental exposure. Since "light" chemical polishing seeks to remove $20 \mu \mathrm{m}$ of metal or less, danger of excessive hydrogen absorption should be low. Moreover, "light" chemical polishing is often applied after cavities have been given a de-gassing anneal. This practice is commonly used as a final step before cavity rinsing, assembly, and evacuation for testing, and its success is not surprising 
based on this study. More importantly, since $<10 \%$ strain is easily achieved by a recrystallization anneal, it could be possible to forego the high-vacuum de-gassing step if cavities are annealed prior to chemical polishing. A very simple and cost-effective cavity procedure might thus emerge: anneal, polish, rinse, test. This sequence inverts the present polishing paradigm.

Vacuum annealing at $800{ }^{\circ} \mathrm{C}$ for 2 hours removed traces of hydrogen and most effects of strain from the $11 \mathrm{~K}$ data. This is consistent with the present wide application of this procedure to de-gas hydrogen from SRF cavities after chemical polishing. However, what may not be fully appreciated is that the full recrystallization of the metal removes dislocations and prevents any assistance to hydrogen uptake for subsequent chemical treatment. Moreover, evidence was found to suggest that hydrogen tends to associate with the dislocation network, so the parameters of the heat treatment might focus instead on removing dislocations instead of de-gassing hydrogen.

Resistance data acquired between 11 and $300 \mathrm{~K}$ showed an increase between 50 and $140 \mathrm{~K}$ for the most heavily polished samples. This change was attributed to formation of small hydride precipitates along the dislocation network, since collection of hydrogen into large hydride precipitates was previously noted to reduce overall scattering in annealed samples. Hydrogen was thought to increase the strain field of dislocation cores above $140 \mathrm{~K}$. The behavior of resistance samples was markedly different from that of RF cavities, in that signatures of hydride precipitates appeared after cooling over minutes, whereas long incubation periods are sometimes required for Qdisease to appear in SRF cavities.

\section{Acknowledgments}

Fermilab is operated by Fermi Research Alliance, LLC under Contract No. DE-AC02-07CH11359 with the United States Department of Energy. The authors would like to thank D.Ford, A Romanenko, F Barkov, and H.Padamsee for stimulating discussions. Chemical work was carried out with the kind assistance of D. Hicks, R. Schuessler, and C. Thompson. Heat treatment work has been aided by A. Rowe, D. Bice and M.Wong.

\section{References}

[1] Adolphsen C et al. 2013 The International Linear Collider Technical Design Report, Vol. 3 - Accelerator, Part I: R\&D in the Technical Design Phase, Section 2.3. http://www.linearcollider.org/ILC/Publications/TechnicalDesign-Report

[2] Schlander F, Aderhold S, Elsen E, Reschke D, and Wenskat M 2011 Quality assessment for industrially produced highgradient superconducting cavities, Proceedings of IPAC2011, San Sebastián, Spain, p. 275, http://accelconf.web.cern.ch/Accelconf/IPAC2011/papers/m opc085.pdf

[3] Weise H 2009 The European XFEL based on superconducting technology, Proc. SRF2009, Berlin, Germany, p. 6,
http://accelconf.web.cern.ch/AccelConf/SRF2009/papers/mo oaau02.pdf

[4] 2005 Standard Specification for Niobium and Niobium Alloy Strip, Sheet, and Plate, ASTM B 393, ASTM International, West Conshohocken PA United States; Cooley L 2011 Technical specifications for high RRR grade niobium sheet for use in superconducting radio frequency cavities, Fermilab Specification 5500.000-ES-371037 Rev B

[5] Kneisel P, Amato J, Kirchgessner J, Nakajima K, Padamsee H, Phillips L, Recce C, Sundelin R, and. Tigner M 1985 Performance of Superconducting Storage Ring Cavities IEEE Trans. Magn. 21 1000; Kneisel P 1999 High gradient superconducting niobium cavities: A review of the present status IEEE Trans. Appl. Supercond. 91023

[6] Saito K, Inoue H, Kako E, Fujino T, Noguchi S, Ono M, and Shishido T 1998 Superiority of Electropolishing over Chemical Polishing on High Gradients Particle Accelerators 60193.

[7] Bieler TR, Wright NT, Pourboghrat F, Compton C, Kartwig KT, Baars D, Zamiri A, Chandrasekaran S, Darbandi P, Jiang H, Skoug E, Balachandran S, Ice GE, and Liu W 2010 Physical and mechanical metallurgy of high purity $\mathrm{Nb}$ for accelerator cavities Phys. Rev. Special Topics - Accel. And Beams 13031002

[8] Bonin B and Röth RW 1992 Q degradation of niobium cavities due to hydrogen contamination, Particle Accelerators 40 59; Antoine CZ, Aune B, Bonin B, Cavedon JM, Juillard M, Godin A, Henriot C, Leconte P, Safa H, Veryssiere A, The role of atomic hydrogen in Q-degradation of niobium superconducting RF cavities: Analytical point of view Op. Cit. 616

[9] Moffat D, Barnes P, Kirchgessner J, Padamsee H, Potts J, Rubin D, Sears J, Shu Q, and Proch D 1991 Increase in RF surface resistance of niobium as a result of acid treatment, Proc. 1991 Particle Accelerator Conference (IEEE Service Center, N.J.) p. 2414

[10] Knobloch J and Padamsee H 1998 Enhanced susceptibility of $\mathrm{Nb}$ cavity equator welds to the hydrogen related Q-virus, Proceedings of the 1997 Workshop on RF superconductivity, Padova, Italy (Palmieri V and Lombardi A ed., INFN, Legnaro), p. 434

[11] Cooley LD, Burk D, Cooper C, Dhanaraj N, Foley M, Ford D, Gould K, Hicks D, Novitski R, Romanenko A, Schuessler $\mathrm{R}$, Thompson C, and Wu G 2011 Impact of forming, welding, and electropolishing on surface finish of niobium coupons and superconducting $\mathrm{RF}$ cavity equator regions IEEE Trans. Appl. Supercond. 212609

[12] Cooley LD, Hahn E, Hicks D, Romanenko A, Schuessler R, and Thompson C 2012 Annealing to mitigate pitting in electropolished niobium coupons and SRF cavities, AIP Conference Proceedings 1435, 289

[13] Manchester FD and Pitre JM 2000 Phase Diagrams of Binary Hydrogen Alloys, ed Manchester FD (Metals Park, $\mathrm{OH}$ : ASM International Publications)

[14] Barkov F, Romanenko A, and Grassellino A 2012 Direct observation of hydrides formation in cavity-grade niobium Phys. Rev. Special Topics-Accelerators and Beams, 15 122001.

[15] Barkov F, Romanenko A, Trenikhina Y, and Grassellino A 2013 Precipitation of hydrides in high purity niobium after different treatments J. Appl. Phys. 114164904

[16] Tao R, Romanenko A, Cooley LD, and Klie RF 2013 Low 
temperature study of structural phase transitions in niobium hydrides Journal of Applied Physics 114, 044306-044306.

[17] Romanenko A, Barkov F, Cooley LD, and Grassellino A 2013 Proximity breakdown of hydrides in superconducting niobium cavities Supercond. Sci. Technol. 26(3) 035003.

[18] Romanenko A and Padamsee H 2010 The role of nearsurface dislocations in the high magnetic field performance of superconducting niobium cavities Supercond. Sci. Technol. 23(4) 045008

[19] Paxton HW, Sheehan JM, and Babyak WJ 1959 Observation on the niobium (columbium)-hydrogen system Trans.Met. Soc. AIME 215 725; Komjathy S 1960 The niobiumhydrogen system J. Less Common Metals 2(6) 466

[20] Pfeiffer G and Wipf H 1976 The trapping of hydrogen in niobium by nitrogen intersttitials J.Phys.F: Metal Phys, 6(2) 167

[21] Flynn CP and Stoneham AM 1970 Quantum theory of diffusion with application to light interstitials in metals Phys. Rev. B 13966

[22] Ford DC, Cooley LD, and Seidman DN 2013 Suppression of hydride precipitates in niobium superconducting radiofrequency cavities Supercond. Sci. Technol. 26 (to appear)

[23] Zabel H and Peisl J 1979 The incoherent phase transitions of hydrogen and deuterium in niobium J.Phys.F:Metal Phys. 9(7) 1461

[24] Williams P, Evans Jr. CA, Grossbeck ML, and Birnbaum HK 1976 Ion microprobe analysis for niobium hydride in hydrogen-embrittled niobium Anal. Chem., 48 (7) 964

[25] Dzyuba A, Romanenko A, and Cooley LD 2010 Model for initiation of quality factor degradation at high accelerating fields in superconducting radio-frequency cavities Supercond. Sci. Technol. 23(12) 125011

[26]Saito K and Kneisel P 2000 Temperature dependence of the surface resistance of niobium at $1300 \mathrm{MHz}$. Comparison to BCS theory, Proc. of 1999 Workshop on RF Superconductivity, Santa Fe, NM, USA

[27] Ciovati G 2004 Effect of low-temperature baking on the radio-frequency properties of niobium superconducting cavities for particle accelerators J. Appl. Phys. 96(3) 1591

[28] Dhakal P, Ciovati G, Myneni GR, Gray KE, Groll N, Maheshwari P, McRae DM, Pike R, Proslier T, Stevie F, Walsh RP, Yang Q, and Zasadzinski J 2013 Effect of high temperature heat treatments on the quality factor of a largegrain superconducting radio-frequency niobium cavity Phys.Rev.Special Topics Accel.Beams 16042001

[29] Romanenko A, Edwardson CJ, Coleman PG, and Simpson PJ 2013 The effect of vacancies on the microwave surface resistance of niobium revealed by positron annihilation spectroscopy Appl. Phys. Lett. 102232601

[30] Grassellino A, Romanenko A, Melnychuk O, Trenikhina Y, Crawford A, Rowe A, Wong M, Sergatskov D, Khabiboulline T, and Barkov F 2013 Nitrogen heat treatments of superconducting niobium radio frequency cavities: a pathway to highly efficient accelerating structures Supercond. Sci. Technol. 26 (to appear).

[31] Casalbuoni S, Knabbe EA, Kötzler J, Lilje L, von Sawilski L, Schmüser P, and Steffen B 2005 Surface superconductivity in niobium for superconducting RF cavities Nucl. Instrum. Meth. Phys. Res. A 538(1) 45-64

[32] Higuchi T and Saito K 2003 Hydrogen absorbtion in electropolishing of niobium AIP Conf. Proc. 671 203; Paul RL, Chen-Mayer HH, and Myneni GR 2003 Determination of hydrogen in niobium by cold neutron prompt gamma-ray activation analysis and neutron incoherent scattering op. cit. 151

[33] Ricker RE and Myneni GR 2010 Evaluation of the propensity of niobium to absorb hydrogen during fabrication of superconducting radio frequency cavities for particle accelerators J. Res. Natl. Inst. Stand. Technol. 115353

[34] Antoine CZ, Berry S, and Shou H 2004 Hydrogen surface analysis of niobium in function of various electrochemical conditions, in Proc. 11th Workshop on RF

Superconductivity, Lübeck/Travemünder, Germany, 2003 (DESY, Hamburg) 596

[35] Higuchi T, Saito K, Yamazaki Y, Ikeda T, and Ohgushi S 2003 Hydrogen Q-disease and electropolishing, in Proc. $10^{\text {th }}$ Workshop on RF Superconductivity, Tsukuba, Japan (edited by Noguchi S et al., KEK, Tsukuba, Japan) 427

[36] Isagawa S 1980 Hydrogen absorption and its effect on lowtemperature electric properties of niobium J. Appl. Phys, 51(8) 4460

[37] Singer W, Ermakov A, and Singer X 2010 RRR measurement techniques on high purity niobium, TTCReport 2010-02 (Tesla Technology Collaboration, DESY, Germany)

[38] Cooley LD 2012 Operational procedures for residual resistivity ratio (RRR) measurement of niobium, Fermilab TD 5220-OP-464080 (Fermilab, Batavia IL USA)

[39] Splett JD, Vecchia DF, and Goodrich LF 2011 A comparison of methods for computing the residual resistivity ratio of high-purity niobium J. Res. Natl. Inst. Stand. Technol. 116489

[40] Sokolenko VI 1999 On the contribution of dislocations to the resistivity of niobium Low Temperature Physics 25(4) 263

[41] Westlake DG 1969 Mechanical behavior of niobium(columbium) - hydrogen alloys Trans. Metall..Soc. AIME 245287

[42] Makenas BJ and Birnbaum HK 1980 Phase changes in the niobium-hydrogen system I: Accommodation effects during hydride precipitation Acta Metall. 28(7) 979

[43] Birnbaum HK and Sofronis P 1994 Hydrogen-enhanced localized plasticity - a mechanism for hydrogen-related fracture Mater. Sci. Eng. A 176(1) 191; Grossbeck ML and Birnbaum HK 1977 Low temperature hydrogen embrittlement of Niobium II-microscopic observations Acta Metall. 25(2) 135

[44] Reuther TC and Aachter MR 1970 Dislocation diffusion in niobium (columbium) single crystals. Metallurgical and Materials Transactions B 1(6) 1777

[45] Love GR 1964 Dislocation pipe diffusion Acta Metallurgica 12(6) 731

[46] Mehrer H 2007 Dislocation Pipe Diffusion, Diffusion in Solids: Fundamentals, Methods, Materials, DiffusionControlled Processes (Springer Series in Solid-State Sciences, vol. 155, Springer Berlin Heidelberg) 583-591

[47] Ford D, Cooley LD, and Seidman DN 2013 First-principles calculations of niobium hydride formation in superconducting radio-frequency cavities Supercond. Sci. Technol. 26095002

[48] Albrecht WM, Goode WD, and Mallett MW 1959 Reactions in the Niobium-Hydrogen System J. Electrochem. Soc. 106(11) 981

[49] Welter JM, and Schondube F 1983 A resistometric and 
neutron diffraction investigation of the $\mathrm{Nb}-\mathrm{H}$ system at high hydrogen concentrations J. Phys. F: Metal Phys. 13(2) 529

[50] Heuser BJ and Chen WC 1999 Effect of lattice defects and temperature transition rates on the deuteride (hydride) particle morphology and phase transformation thermal hysteresis in niobium J. Phys.: Condens. Matter 11(38) 7195.

[51] H.K.Birnbaum et al., "Hydride precipitation in $\mathrm{Nb}$ and some properties of NbH", J.L-Com Met. 49(1976).

[52] J.A.Rodrigues and R.Kirchem, "More evidence for the formation of a dense cottrell cloud of hydrogen (hydride) at dislocations in niobium and palladium", Scr.Met. 17, pp.159-164(1983)

[53] Pundt A and Kirchheim R 2006 Hydrogen in metals: microstructural aspects. Annu. Rev. Mater. Res. 36555

[54] Koike H, Shizuku Y, Yazaki A, and Fukai Y 2004 Superabundant vacancy formation in $\mathrm{Nb}-\mathrm{H}$ alloys; resistometric studies J. Phys.: Condens. Matter 16(8) 1335

[55] Cížek J, Procházka I, Bečvář F, Kužel R, Cieslar M, Brauer G, Anwand W, Kirchheim R, and Pundt, A 2004 Hydrogeninduced defects in bulk niobium Phys. Rev. B 69(22) 224106; 2005 Hydrogen-induced defects in niobium studied by positron annihilation spectroscopy J. Alloys Comp. $\mathbf{4 0 4}$ 580

[56] Shi SQ and Li WB 1985 Studies of hydrogen trapped by nitrogen or oxygen in niobium with ultrasonic attenuation technique and resistivity measurements Le Journal de Physique Colloques 46(C10) C10-91 